\title{
Creating Dynamic Groups using Context-awareness
}

\author{
Josef Hallberg \\ Luleå University of Technology \\ SE-971 87 Luleå \\ josef.hallberg@Itu.se
}

\author{
Mia Backlund Norberg \\ Luleå University of Technology \\ SE-971 87 Luleå \\ mia.backlund- \\ norberg@ltu.se
}

\author{
Johan Kristiansson \\ Ericsson Research \\ SE-971 28 Luleå \\ johan.j.kristiansson@ \\ ericsson.com
}

\author{
Kåre Synnes \\ Luleå University of Technology \\ SE-971 87 Luleå \\ kare.synnes@ltu.se
}

\author{
Chris Nugent \\ School of Computing and \\ Mathematics \\ University of Ulster \\ N.Ireland \\ cd.nugent@ulster.ac.uk
}

\begin{abstract}
This article presents the conceptual communication model of dynamic groups, that dynamically utilizes three traditional communication metaphors through the use of contextbased information. Dynamic groups makes creation, management and usage of groups easy. It enables social network structures to be maintained in both virtual and faceto-face settings as well as in the combination thereof. This article defines the dynamic management of advanced contact lists which can include presence and status information, a/synchronous multimedia communication tools, and methods for structuring social networks. It also contains an initial evaluation and a proposed architecture for technichal realisation.
\end{abstract}

\section{Categories and Subject Descriptors}

I.2.8 [Artifical Intelligence]: Problem solving, control methods and search - Heuristic methods

\section{General Terms}

Design

\section{Keywords}

Social networks, contact list, presence

\section{INTRODUCTION}

It is no longer self-evident that communication should take place face-to-face. Many choose to maintain virtual social networks, through the use of internet tools, as well as their normal social networks with friends, colleagues, etc. The availability that the Internet provides has made it possible

Permission to make digital or hard copies of all or part of this work for personal or classroom use is granted without fee provided that copies are not made or distributed for profit or commercial advantage and that copies bear this notice and the full citation on the first page. To copy otherwise, to republish, to post on servers or to redistribute to lists, requires prior specific permission and/or a fee.

MUM'07, December 12-14, 2007, Oulu, Finland.

Copyright 2007 ACM 978-1-59593-916-6/07/0012 ...\$5.00. to find and communicate with people all over the world [4]. While these internet tools allow people to meet and share experience in new ways, they are not always easy to use, especially not in mobile terminals with limited input capabilities. Using them can also result in an increased amount of management and stress. This is particularly true for conferencing communication tools or the next-generation multimedia telephony system [6] where users have to manually invite all participating users to establish a session. For example, users are typically required to remember multiple addresses like SIP URIs or phone numbers to other users, or alternatively manually selecting them from a personal friends list, assuming the users can be found there.

This paper outlines a model based on context-awareness to aid setting up group communication sessions. Imagine a communication system where a user can form groups effortlessly and on the user's terms with minimal user interaction. Groups can be formed whenever and wherever the user chooses, be it for over a day, or for a longer period of time. Using the proposed model, a dynamic group can either be formed manually between people who are co-located, by joining a desired group, or by a user who invites others (either by searching, or picking from a list), or automatically based on predefined context-aware rules. The authors believe that dynamic groups can greatly simplify the way people communicate and cooperate in groups.

The main focus of this article is to define the dynamic group model (e.g. how to find users, form and maintain groups, and support context sharing) and not go into details about network and network protocols. There are a number of research questions related to dynamic groups:

- How should dynamic groups be formed and maintained?

- How should sensor-data and contexts be stored to support the functionality of dynamic groups?

- How should rules for automatic establishment of dynamic groups be created?

- What should a profile system contain to provide the functionality of the dynamic groups? 


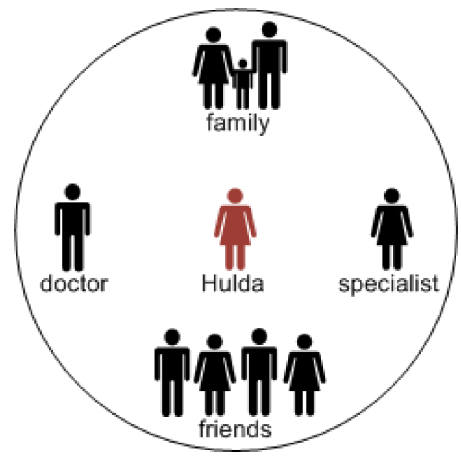

Figure 1. A user-centric view of dynamic groups

To illustrate the advantages of dynamic groups we present two different scenarios. One scenario is from a healthcare perspective where the priority for simplicity and automaticity motivate the use of smart environments in conjunction with decision support rules for creating dynamic groups. The other is from a normal every-day-use perspective where a user simply might want to uphold his/her virtual social network in a simple manner.

\subsection{Scenario 1 - Healthcare}

Hulda is 77 years old and is still living at home. She uses the dynamic group system in order to maintain social relations. With this tool Hulda has easy access to her friends, family, and healthcare professionals (see Figure 1). In addition to communication, the system which Hulda uses has some context-aware rules built in for medical reasons, as well as calendar functions to remind her of her social events.

Hulda always start her day with a cup of tea in her living room. She has requested that the dynamic groups system automatically connects her to others who are currently having their morning tea or breakfast. The living-room sofa is equipped with sensors and detect when Hulda sits down, and a query to the profile database for "current activity: breakfast" and/or any public group with the keyword "breakfast" is issued and Hulda gets connected.

After Hulda has had her tea she prepares for her morning exercise. Her smart clothing is equipped with a number of health monitoring sensors, which can measure her ECG among other things. This morning Hulda gets more exhausted than normal, and this is detected by the system which automatically contacts the private healthcare group and puts Hulda in contact with the house doctor who can help her.

\subsection{Scenario 2 - Normal Every-day-use}

Anna is a teenage girl living at home with her parents. She is an active girl who likes to hang out with her friends, arrange parties and go horseback riding. This Tuesday, she meets up with a couple of friends downtown, where they discuss what to do on Friday evening. Anna proposes they should have a party at her house, though she has to check with her mum and dad first. She picks up her dynamic groups enabled mobile phone and creates a protected group with the friends she's meeting with. Later that night, after she checked with her parents, she sends a text-message to the group to let her friends know the party is on.

On Thursday night Anna goes horseback riding. Once in the stable she decides to invite three of her stable friends to the party and invites them to the dynamic group. Anna notices a few more people have been invited to the dynamic group as the party-group is always up-to-date for all participants.

After the party, and after all the funny photos and videos from the party have been shared with the group, the partygroup fades away quickly (i.e. is not presented on top to the members) since the party is over and the group is no longer relevant. However, this party group has been added to Anna's social network so she (or any of the other group members) can easily recreate the same dynamic group if she decides to have another party in the future.

\subsection{Outline of the Document}

The rest of the paper is organized as follows: Section 2 introduces the dynamic group model and explains the concept. In Section 3 some implementation issues are discussed. Section 4 covers privacy and security issues. In Section 5 a short user evaluation is presented. The discussion is covered in Section 6 while conclusions and future work is covered in Section 7.

\section{THE DYNAMIC GROUPS MODEL}

There are currently several tools available for group communication, cooperation, and organization. Often these tools require users to know details about the contact they wish to reach. There are a number of ways in which people choose to find the people they want to communicate with:

- The room metaphor: The classic room metaphor is where the user simply joins a room focusing on the topic of interest. The tools range from advanced tools such as group conferencing software [15, 21, 28] which allows for several media (e.g. video, audio, chat, etc.), to live chat groups [11] and even simple web-forums [23].

- The friend metaphor: Many of the popular friend metaphor tools today provide a simple search function which lets the user search for friends by username or contact details. The user can then add contacts to their "friends list" and then communicate with them. The tools started out as simple instant messaging applications $[1,10]$, but some have evolved and now also provide more advanced functions such as the possibility to send audio and video $[16,26]$.

- The tree metaphor: Some tools exist mainly for mapping social networks and can be categorized as tree metaphor tools. While some of these tools provide communication functionality $[17,20]$ the main functionality is to provide the user with an overview of their social network and show how the user fits in as a node in a larger tree structure. Relations to other people can often be several different things, such as family member, friend, or colleague. Some of these tools also provide advanced search capabilities which helps the user find other people $[2,14]$.

The authors seek to define a new conceptual communication model which interweave the three traditional commu- 
nication metaphors dynamically, in a user friendly way. The definition of the dynamic group model is as follows:

"The dynamic group model defines group communication where the participants can be invited based on context and social networks in both physical and virtual settings."

The model has many similarities to existing solutions and can be used in pretty much the same way. However, a dynamic group offers new functionality such as the possibility for automatic group formation based on predefined rules. Simply put, the dynamic group model is a combination of all three metaphors, namely the room metaphor, the friend metaphor, and the tree metaphor. The user can maintain a friends list in parallel to groups, groups can be created much like rooms, and the history of past groups can help build an understanding of relations between users.

\subsection{Forming and Maintaining Groups}

Groups can be formed in many different ways. Co-located people could form a group easily by using Bluetooth or Near Field Communication on the currently used device, or it could be done manually by simply adding people to a group. A user could also form a group by organizing contacts and making groups to simplify tasks for him-/herself. Finally, a group can be formed which will be open for other users to join, such a group could be a discussion group, or simply an interest group focusing on a specific topic. Since groups can be formed in a variety of ways the authors have defined three main types of groups, namely Private, Protected, and Public:

- Private groups - the main purpose for a private group is to help organize contacts for a user. The private group is not visible to anyone else, only to the user. Group members of a private group do not know they are in this private group, nor do they know of each other. A simple use for a private group could be to form a temporary group to which a user could send vacation photographs or small messages.

- Protected groups - the main purpose for protected groups is to make it easy to create secure groups between people who know of each other. All participants in the group can be seen as administrators and are able to invite other people, however the group is not searchable and it is not possible to join the group apart from being present when the group is formed or by getting an invitation from a member of the group. Protected groups are useful when friends meet and decide to organize an event later on, or for co-workers who decide to follow up on a meeting.

- Public groups - the main purpose for public groups is to provide an open forum where things can be discussed. The creator of the group automatically gets the administrative role, but can assign administrative powers to other group members. A user can search for public groups based on keywords which can be the topic of the group, and join them. An example of a public group could be a public blog, or a group discussing a specific topic together.
Dynamic groups rely on an advanced user profile system where specific information about the user is listed. Such information can be user id, user name, other contact details, interests, expertise, and current location. This stored data is similar to what many currently available tools use, with the main difference being that this data is used to organize the contact list. To talk to a friend, or a number of friends, the user can simply select the contact(s) from a list, or search the contact list for specific criteria (e.g. friends who are interested in cars). To find a new contact the user can simply search the profile database for the desired criteria (e.g. people with interest in cars, or experts in computer science). However, there is no need to perform a query if the users are co-located as mentioned earlier in this section.

Once a group becomes inactive it fades out from the user interface, but the information about the group is not removed unless the user explicitly removes it. Groups which have faded away can be recalled by searching or browsing for it. For private groups only the creator can recall it, for protected groups only those once part of it can recall it, and for public groups which have not explicitly been removed anyone can search for it. This history function helps maintain groups, as well as social networks.

\section{IMPLEMENTATION}

Dynamic groups require several technical solutions to work properly. This section discusses the most essential parts required together with related work for each part. For storing contexts the authors propose to use HomeML[19], an Extensible Markup Language (XML) standard for sharing data between smart environments. For handling rules to provide automaticity the users propose to use HomeRuleML[8], a rule engine which has been integrated with HomeML. Finally, to handle the profile system as well as query requests and results, the authors propose to develop HomeCom which will be integrated with HomeML as well.

\subsection{Context-platform}

The purpose of the context-platform is to store contexts from sensors in the environment. Some of the data, such as location data, is used in certain queries such as "start a dynamic group with the people close to me". Other data is needed to make it possible to make automatic decisions regarding the forming of dynamic groups. The scenario in Section 1.1 uses several contexts for its operation, such as the activity context "drinking tea", the time context "morning", and the location context "sitting on living-room sofa" to make the decision to create/join a dynamic "breakfast" group. Later on in the same scenario there is an example of how healthcare data is used for contacting healthcare professionals.

The context-platform used for dynamic groups needs to be flexible and easy to configure and use in several different terminals. XML [30] offers such flexibility as it is an acknowledged standard for sharing of data over the Internet. Using XML as a front-end for sharing data between context-server and clients hides the complexity of the back-end implementation. Context Fabric (confab) [9] is one of the contextplatforms which uses XML for the handling of data. Confab also includes an elaborate solution for handling of privacy issues, which unfortunately also makes the protocol quite 


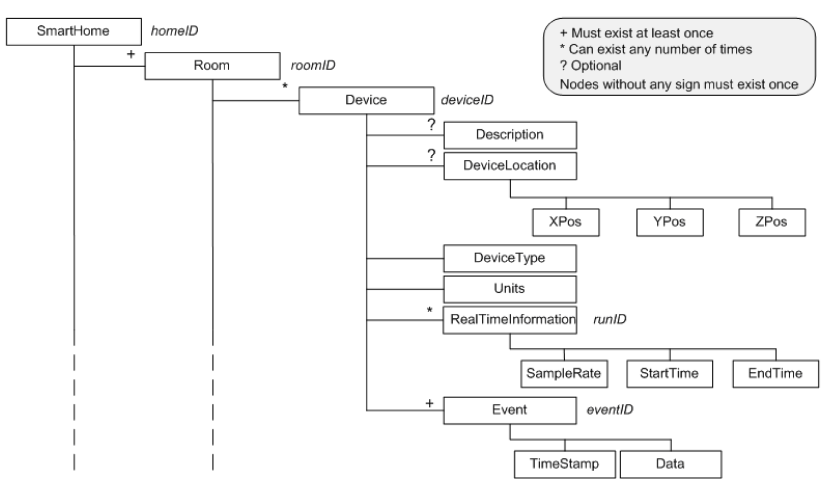

Figure 2. Excerpt of the HomeML definition

complex and difficult to configure and update.

Apart from Confab there are several other available contextplatforms. These include Context Toolkit [5], Aware Home [12], Gaia [24], Aura [27], etc. These platforms were developed specifically for smart environments to provide contextaware services. However, they do not provide the flexibility that XML offers and make less suitable candidates for dynamic groups.

For dynamic groups the authors propose the use of HomeML [19] an XML standard for sharing data between smart environments, see figure 2. This is an open standard for which researchers can propose changes and develop tools. One of the benefits of using HomeML for dynamic groups is that the standard is expected to be deployed in many smart environments around the world. Hence, this should simplify the integration of dynamic groups in the existing smartenvironments. Additionally, several useful tools are being developed for this standard, which can be used to improve the functionality of dynamic groups.

\subsection{Rule-engine}

In a fixed smart-environment it is often possible to predevelop rules to function in the environment. This is true especially if the developers have knowledge of, or can control, which inhabitants will use the environment, their behaviour, etc. Dynamic groups however are meant to be open and dynamic, with the possibility of being customized to the individual who will use it. This means that the rules must be easy to create and customize for each and every individual, and it is also desirable to make editing rules so simple that the users can do this themselves. An interesting use of a rule engine is to add the possibility to have invitations show up on the terminal a user is active on, and to determine which communication media to use (e.g. video, sound, chat, etc.). The algorithm presented in "Supporting Automatic Media Resource Selection Using context-awareness" [13] can be used to achieve this.

Many smart environments use rule-based systems for their logic handling $[24,27]$ but these are not very suitable for a general solution such as dynamic groups. Instead we turn to HomeRuleML [8], an open XML model (Figure 3 for representing rules which are meant to function together
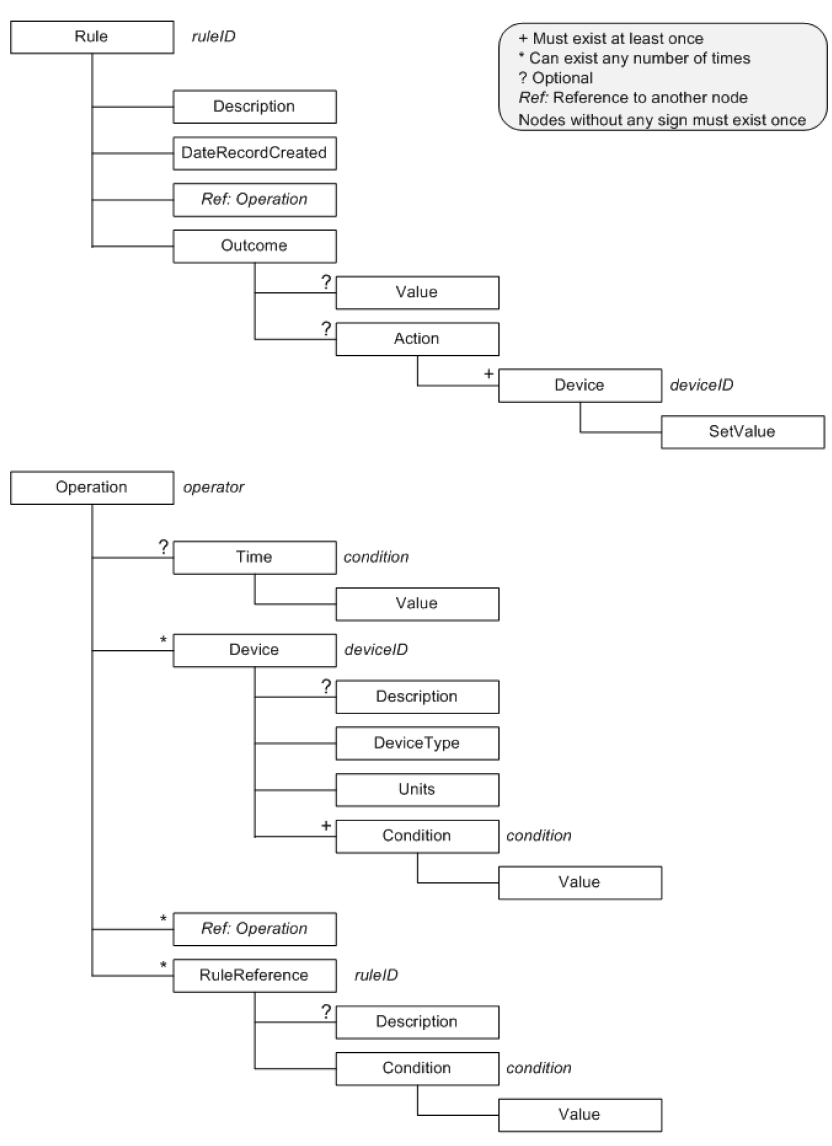

Figure 3. The HomeRuleML definition

with HomeML. Since this is an open standard it is likely several tools will be developed which will help users develop advanced rules in a simple manner. Two graphical front-end concept tools, HomeCI [18] and HomeTL [25], for HomeRuleML already exist and further development of these might prove to be very useful for developing rules to dynamic groups.

HomeRuleML uses sensor- and context-data which has been stored in a HomeML enabled smart environment. This means that HomeRuleML rules can focus on specifying the rules by defining which sensors and contexts are of interest, instead of dealing with the data retrieval etc. The model is recursive which enables advanced logic expressions and the reuse of other, more generic, rules. Common logical operators (AND, OR, NAND, NOR, XOR, NOT, and a special operator $O R D E R$ ) are being used to form the expressions, and common condition operators (greater than, less than, equals, not equals) are used to set calculate conditional expressions.

\subsection{Profile-system}

Typically a profile system contains information such as address, telephone number, interests, etc. In most of these systems $[10,16,26]$ it is not possible to query for users with specific information, the information serves no other purpose than to inform friends etc. Instead users add people to their friends list based on a user id. This works ok when 
adding people already known to a user, but not so well when trying to establish new contacts. What is needed in dynamic groups is a profile system which lets a user search for people based on several contexts and information.

Further work has also been done in trying to find which people know what, etc. IKNOW [3] is a communityware tool which makes it possible for its users to search the network for answers to questions such as "who knows what?" and "who knows who knows what?". This is a powerful tool but also complex, which defeats the purpose of a simple profile system that can be easily queried for contacts.

The authors propose a new model for representing user profiles called HomeCom. This model will be based on XML, similarly to HomeML and HomeRuleML which has previously been mentioned. This model will build on both the context-platform and the rule-engine to form a system in which a user can search for people based on the present information. The HomeCom model helps users manage groups and provide invite, add, remove, and query functionalities. It simplifies data handling to and from the HomeML server. Using XML for a profile system has several benefits. The hierarchical nature of XML makes it very suitable for representing user profiles. It also enforces a specific structure which in turn opens up for graphical user-interface developers to develop compatible clients for dynamic groups.

There are of course some bandwidth concerns if there are several users querying for contacts using dynamic groups. One solution to this problem would be to use mirror servers. This could offer necessary backup, as well as load balancing. During initial tests the system can use one central server, and as dynamic groups are more accepted and used it can be extended with more servers. Of course these servers also need to incorporate necessary security solutions to prevent malicious users from altering the system and other users' information.

\subsection{Session Management in Clients}

Integrating the proposed model with an existing communication tool or service can be done in various ways as long as the underlying system has support for group communication. To give an example of how the dynamic group model can be used, this section briefly describes how it can be integrated with a SIP-based communication system. To handle group communication many SIP-based systems use a centralized server running in the network to duplicate and route RTP streams to each other client. Briefly put, implementing conferencing can be done in two different ways [7]:

- Dial-in - dial-in is based on letting each participating user send a SIP Invite message to the conference server in order to be part of the session. Users can also invite other users by sending a SIP Refer message asking them to join the conference.

- Dial-out - dial-out lets the conference server invite all participating users by sending a SIP Invite message directly to them. In this case, to invite users to a session, the users typically sends a SIP Invite to the conference server containing a list of all other participating users.

In both the dial-in and dial-out case, some user needs to maintain a list of all participating users in order to in-

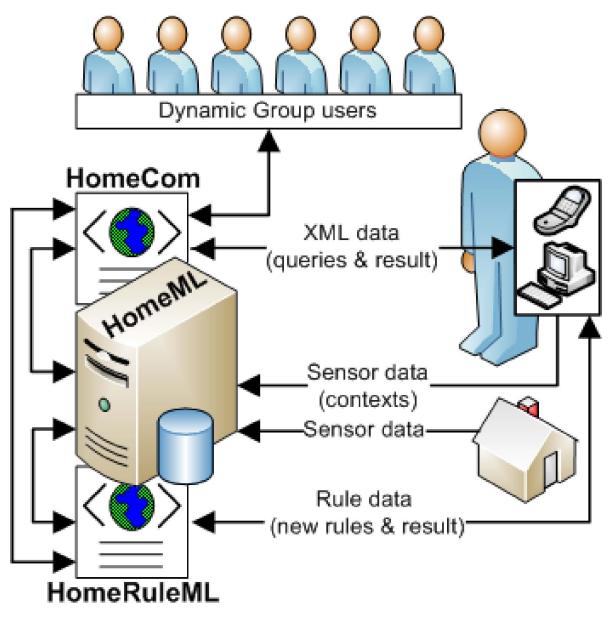

Figure 4. An overview of the parts which form the Dynamic Group system

vite them to the conference session. The proposed dynamic group model can thus be implemented by providing such a list to the clients. A list could for example be sent to the call control module which is a part of most clients.

\subsection{Architectural Overview}

This section has described the essential parts of a dynamic groups architecture, as seen in Figure 4. Section 1.1 describes a smart environment which reports sensor data so the system can assist the user in every day life. This is combined with the ability to form dynamic groups with family, friends, and healthcare personnel. The sensors which are located in both user-carried mobile devices and the environment report data to the HomeML server. The HomeRuleML model listens to new data and checks the data according to the predefined rules.

In the example when the user sits down in the living-room sofa the rule stored in HomeRuleML triggers. An XML query is sent to HomeCom containing a logical expression (e.g. "Breakfast AND Senior Citizens"). HomeCom parses the expression, searches the database, and returns available groups which match the query. By default, the groups are presented in order of activity with the most active group first, however the result can be sorted using other criteria. The rule is set to join the first group in the result list.

In Section 1.2 an everyday scenario is described. Initially the user wants to form a group in order to plan a party. The user can form this group in a number of different ways, for instance by using built in Near Field Communication or Bluetooth, or even by selecting the members from a list. The user can also query for other people by sending a request to the HomeCom model.

\section{PRIVACY AND SECURITY}

Privacy is an important factor when it comes to contextplatforms, and profile systems. While there are solutions which consider this problem, such as Confab [9], the needs are slightly different in a dynamic groups architecture. Of course healthcare data needs to be unavailable to the pub- 
lic. It should also be possible for a user to choose which information to store in the context-platform.

Pentland [22] has discussed ways of dealing with privacy issues in systems similar to dynamic groups. In order to deal with privacy concerns with information, two different solutions are proposed. Either all information is stored, and the user simply selects which information to make public afterwards, or the user can press a "mute"-button to prevent that information being made public. The authors argue that a user may choose to display different information for different groups, however, if a user chooses not to display certain information then they should not have access to similar information from others in the group.

Security is also an important factor in dynamic groups. A user's profile should be updated by the user only, and not by others. A user's social network structure also needs to be secure from tampering as it contains information about user habits etc. Furthermore, some groups, such as public groups, may need to be moderated to make sure information is not spread to unwanted people and to minimize malicious behaviour. Furthermore it could also be possible for the creator of a group to define more fine grained access control where invitation rights etc. can be limited. Further research needs to be done in both security and privacy issues for dynamic groups.

\section{USER EVALUATION}

To illustrate the functionality of dynamic groups the authors developed a simple prototype. The prototype consists of an interactive "paper-prototype" [29] which has the look and feel of a real prototype as seen in Figure 5, but only static content. The prototype shows how groups can be formed, how to add and remove contacts, which contexts a single user can provide, and also a map which can display the location of the users in a group, or a single user.

Qualitative semi-structured interviews ${ }^{1}$ were held with 10 people ( 5 women and 5 men) between the ages of 21 and 53 (mean 34) with average or higher computer experience. The general opinion was that the concept would be quite usable though a few concerns like integrity and information overload were revealed. The results from the user evaluation has been divided into 4 different topics:

- Usage - While there was some initial scepticism amongst a few of the participants they did have some suggestions to what they would use the system for. The usage ranged from leisure activities such as organizing events and meeting with friends, to security applications such as keeping contact with members within hunting parties, to professional applications such as organizing meetings etc. Some concerns were also raised regarding if the system would be wide spread enough upon deployment. In general the major benefit was thought to be the ability to contact a number of people at once.

- Usability - The majority of the participants were quite satisfied with interface and usability aspects.

\footnotetext{
${ }^{1}$ The results from the full interview can be found at
} http://media.csee.ltu.se/projects/mymates/

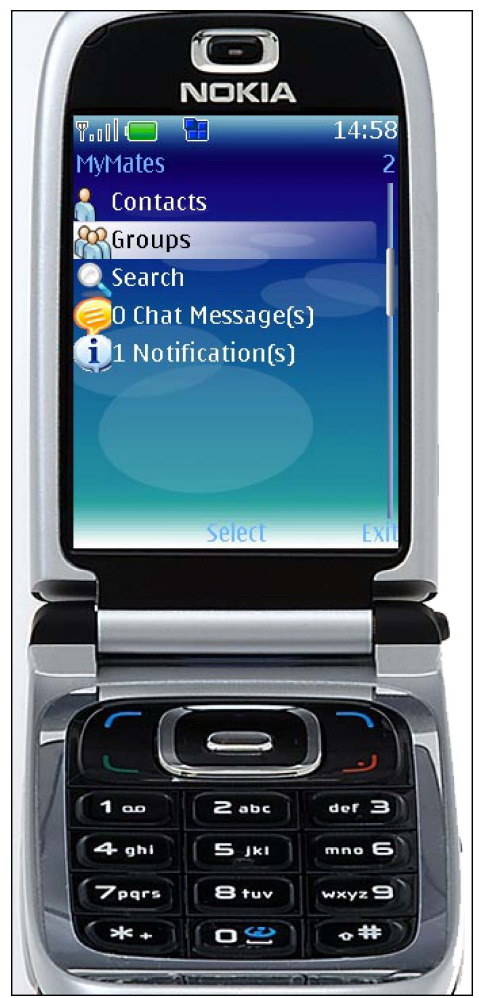

Figure 5. Screenshot of the prototype

However, one comment was raised regarding the content switching between screens, or more specifically that the layout and options changed. One person also commented on the quality of the graphics, and that these should be improved for the final product.

- Functionality - Over the brief demonstrations there were few comments about additional functionality requirements or change of functionality. One comment was to add control over which media to be contacted on, such as choosing only to be contacted through chat messages from a specific group. Other comments had to do with interface design, and how to make active contacts and groups more easily accessible. The suggestion was to let inactive contacts and groups fade away (though accessible through scrolling down etc.). Finally, there was a desire to integrate the dynamic groups prototype with existing tools such as the contact lists and instant messaging applications.

- Privacy - Several of the participants had privacy concerns with using the prototype. Many thought that the contexts being shared for public view could be misused by others. There was also some concern for sharing information in protected groups as an undesired person could get hold of a group member's device and through that gain access to the information.

\section{DISCUSSION}

Dynamic groups is a new concept developed by the authors. In this article the model has been described, and the major parts have been discussed. This section discusses the research questions presented in Section 1. 
How should dynamic groups be formed and maintained? Three different methods have been proposed for forming groups, private, protected, and public groups. Private groups are for a single user and are meant to help organize contacts, protected groups are mainly for co-located people (with the option of inviting additional people) which might want to maintain communication after they part, and public groups are for open groups which should be visible to, and searchable by, the general public. If a group is inactive for a while it will fade away, but users can maintain their groups by recalling groups from the history.

How should sensor-data and contexts be stored to allow the functionality of dynamic groups? The authors argue that an open standard based on XML is the best choice for storing sensor-data and contexts for dynamic groups. Hence it has been decided to use HomeML which is developed for storing data from smart-environments, and the authors believe this can be updated to encompass the information necessary for dynamic groups. Because it is an open standard the authors believe useful tools will be developed for handling HomeML data, furthermore using an open standard will help give dynamic groups the impact it needs.

How should rules for automatic establishment of dynamic groups be created? Dynamic groups can be created based on user queries or based on previous constellations. A user can select an advanced query string which will be passed onto the database. The result will be given in form of an XML file which can be parsed and viewed with whatever user interface being used. Creating a dynamic group should be a simple process, and where there are predefined rules it can even be an automatic process. As for automatic generation of dynamic groups the authors propose to use HomeRuleML which is an open XML model for creating rules, it also works together with HomeML.

What should a profile system contain to provide the functionality of the dynamic groups? The authors propose to develop a new model called HomeCom for handling user profiles. This model will be an open XML model similar to HomeML and HomeRuleML already being proposed for dynamic groups. In addition to user id, the model will also contain information such as contact-details, location, interest, expertise, etc. The user may simply choose not to share information which will present an unacceptable privacy risk to the user.

\section{CONCLUSIONS AND FUTURE WORK}

The authors have presented a new model for group communication management called dynamic groups. This model provides advanced functionality for creating and maintaining a virtual social network, and also provides advances querying capabilities for connecting a user to desired people. Dynamic groups are intended for use in every-day-life, be it a healthcare environment, work, or spare-time activities. In addition an evaluation of the model has been performed on 10 people. The results from the evaluation has been positive, although a few concerns regarding privacy and information overflow were raised.

The authors intend to further develop the dynamic group ideas, and refine the user requirements based on feedback from the evaluation. This includes implementing the HomeCom profile system, make the necessary functions for HomeRuleML and HomeML, and continue developing a working prototype for dynamic group systems. For the future prototype the authors also intend to incorporate leading solutions for information fusion in order to derive higher level contexts for use in HomeRuleML. Furthermore the authors wish to investigate if groups can be created autonomously by matching profiles and using ontologies. Finally the prototype will then be evaluated and the results, as well as developed schemas will be made available online to further the impact of dynamic groups.

\section{ACKNOWLEDGEMENTS}

This work has been partly supported through the MyMates Project and the Laboranova project. The authors would also like to thank Stefan Håkansson and Tommy Arngren at Ericsson Research in Luleå, as well as Arne Gylling and Johan Bengtsson at Centre for Distance-Spanning Technology (CDT), for helpful input. Finally the authors would like to thank Zaheer ul Hussain Sani for the work on the prototype.

\section{REFERENCES}

[1] AIM. http://www.aim.com, October 2007.

[2] Classmates. http://www.classmates.com, October 2007.

[3] N. Contractor, D. Zink, and M. Chan. IKNOW: A Tool to Assist and Study the Creation, Maintenace, and Dissolution of Knowledge Networks. In Sunbelt Social Networks Conference, 1998.

[4] C. Dewes, A. Wichmann, and A. Feldmann. An analysis of Internet chat systems. In $I M C$ '03: Proceedings of the 3rd ACM SIGCOMM conference on Internet measurement, pages 51-64, New York, NY, USA, 2003. ACM Press.

[5] A. K. Dey, D. Salber, and G. D.Abowd. A Conceptual Framework and a Toolkit for Supporting the Rapid Prototyping of Context-Aware Applications. Anchor article of a special issue on context-aware computing in the Human-Computer Interaction (HCI) Journal, 16:97-166, 2001.

[6] D. Enstrm, A. Nohlgren, H. Olofsson, J. Peisa, and P. Synnergren. Multimedia telephony for IMS Interoperable VoIP with multimedia support. In Ericsson Review No. 2, 2007.

[7] A. J. et al. Session Initiation Protocol (SIP) Call Control - Conferencing for User Agents (RFC 4579). In $R F C$ 4579, 2006.

[8] J. Hallberg, C. Nugent, R. Davies, K. Synnes, M. Donnelly, D. Finlay, and M. Mulvenna. HomeRuleML - A Model for the Exchange of Decision Support Rules Within Smart Environments. In 3rd annual IEEE Conference on Automation Science and Engineering (CASE), pages 513-520, 2007.

[9] J. I. Hong and J. A. Landay. An architecture for privacy-sensitive ubiquitous computing. In MobiSYS '04: Proceedings of the 2nd international conference on Mobile systems, applications, and services, pages 177-189, New York, NY, USA, 2004. ACM Press.

[10] ICQ. http://www.icq.com, October 2007. 
[11] Internet Relay Chat Protocol. http://www.ietf.org/rfc/rfc1459.txt, October 2007.

[12] C. D. Kidd, R. Orr, G. D. Abowd, C. G. Atkeson, I. A. Essa, B. MacIntyre, E. D. Mynatt, T. Starner, and W. Newstetter. The Aware Home: A Living Laboratory for Ubiquitous Computing Research. In Proceedings of the Second International Workshop on Cooperative Buildings, Integrating Information, Organization, and Architecture, pages 191-198. Springer-Verlag, 1999.

[13] J. Kristiansson, J. Hallberg, S. Svensson, K. Synes, and P. Parnes. Supporting Automatic Media Resource Selection Using Context-Awareness. In In 3rd International Conference on Advances in Mobile Multimedia (MoMM2005), pages 271-282, September 2005.

[14] LinkedIn. http://www.linkedin.com, October 2007.

[15] S. McCanne and V. Jacobson. vic : A Flexible Framework for Packet Video. In ACM Multimedia, pages 511-522, 1995.

[16] MSN. http://www.msn.com, October 2007.

[17] Myspace. http://www.myspace.com, October 2007.

[18] C. Nugent, R. Davies, J. Hallberg, M. Donnelly, K. Synnes, M. Poland, J. Wallace, D. Finlay, M. Mulvenna, and D. Craig. HomeCI - A visual editor for healthcare professionals in the design of home based care. In 29th Annual International Conference of the IEEE Engineering in Medicine and Biology Society (EMBC), pages 2787-2790, 2007.

[19] C. Nugent, D. Finlay, R. Davies, H. Wang, H. Zheng, J. Hallberg, K. Synnes, and M. Mulvenna. homeML An Open Standard for the Exchange of Data Within Smart Environments. In 5th International Conference On Smart homes and health Telematics (ICOST), pages 121-129, 2007.
[20] Orkut. http://www.orkut.com, October 2007.

[21] P. Parnes, K. Synnes, and D. Schefstrm. mSTAR: Enabling Collaborative Applications on the Internet. IEEE Internet Computing, 4(5):32-39, 2000.

[22] A. Pentland, T. Choudhury, N. Eagle, and P. Singh. Human dynamics: computation for organizations. Pattern Recogn. Lett., 26(4):503-511, 2005.

[23] phpBB. http://www.phpbb.com/, October 2007.

[24] M. Romn, Christopher, K. Hess, R. Cerqueira, A. Ranganathan, R. Campbell, and K. Nahrsted. Gaia: A Middleware Infrastructure to Enable Active Spaces. IEEE Pervasive Computing, pages 74-83, Oct-Dec 2002.

[25] A. Rugnone, C. Nugent, M. Donnelly, D. Craig, E. Vicario, C. Paggetti, and T. Tamburini. HomeTL: A Visual Formalism, Based on Temporal Logic, for the Design of Home Based Care. In 3rd annual IEEE Conference on Automation Science and Engineering (CASE), pages 747-752, 2007.

[26] Skype. http://www.skype.com, October 2007.

[27] J. P. Sousa and D. Garlan. Aura: an Architectural Framework for User Mobility in Ubiquitous Computing Environments. In WICAS3: Proceedings of the IFIP 17th World Computer Congress - TC2 Stream / 3rd IEEE/IFIP Conference on Software Architecture, pages 29-43, Deventer, The Netherlands, The Netherlands, 2002. Kluwer, B.V.

[28] Teamspeak. http://www.goteamspeak.com/, October 2007.

[29] The Dynamic Groups Prototype. http://media.csee.ltu.se/projects/mymates/, October 2007.

[30] Extensible Markup Language (XML). http://www.w3.org/XML/, October 2007. 